Año 18, N 48, San Salvador, El Salvador, Centroamérica. Revista Semestral Julio-Diciembre 2018

YEAR 18, N 48, SAN SALVADOR, EL SALVADOR, CENTRAL AMERICA. SEMESTRAL JOURNAL JuLY-DECEMBER 2018

\title{
Contribución de las instituciones de educación superior de El Salvador al aseguramiento de acceso igualitario de hombres y mujeres a una formación técnica, profesional y superior de calidad
}

\author{
Contribution of El Salvador higher education \\ institutions to the insurance of the equal access of all \\ women and men to a quality technical, vocational and \\ higher education
}

William Rafael Rebollo Alvarado Magíster en Gestión y Políticas Públicas de la Universidad de Chile Coordinador del Observatorio de Políticas Públicas de la Facultad de Ciencias Jurídicas UFG. wrebollo@ufg.edu.sv

Fecha de recepción: 28 de septiembre de 2018 Fecha aprobación: 28 de noviembre de 2018

\section{RESUMEN}

La Agenda 2030 para el Desarrollo Sostenible aprobada en Nueva York en septiembre del año 2015, representa un gran logro como un acuerdo internacional que se enfoca en las personas, el planeta, la prosperidad, la paz y las alianzas. Son 17 los objetivos desglosados en 169 metas cuyo logro requiere no solo de la acción de los Estados, sino que de la acción conjunta de actores tanto públicos como privados. Es en ese sentido que, en el presente estudio se aborda el rol de las instituciones de educación superior (IES) de El Salvador respecto a los ODS, específicamente en relación 'con el objetivo 4 de garantizar una educación inclusiva, equitativa y de calidad y promover oportunidades de aprendizaje durante toda la vida para todos, y de manera puntual en el cumplimiento de la meta 4.3 que consiste en asegurar al año 2030 el acceso igualitario de todos los hombres y las mujeres a una formación técnica, profesional y superior de calidad, incluida la enseñanza universitaria, ello, con el objetivo de verificar la contribución de las IES al logro de dicha meta.

Para ello, se aplicaron herramientas de análisis cuantitativo a datos obtenidos del documento Resultados de la Información Estadística de Instituciones de Educación Superior 2016. Se utilizó estadística descriptiva sobre las series de tiempo del período 
2012-2016 para determinar la tendencia respecto a la inscripción de estudiantes, desagregándolos además en razón del sexo, y también, se aplicó el índice de paridad entre los géneros (IPG) sobre los datos de dicho período.

Palabras clave: acceso a la educación, objetivos de desarrollo sostenible, educación superior.

\section{ABSTRACT}

The 2030 Agenda for Sustainable Development approved in New York in September 2015 represents a great achievement as an international agreement that focuses on people, the planet, prosperity, peace and alliances. There are 17 objectives broken down into 169 goals whose achievement requires not only the action of States, but also the joint action of both public and private actors. It is in this sense that the present study addresses the role of higher education institutions (HEIs) in El Salvador with respect to the SDGs, specifically in relation to objective 4 to ensure an inclusive and equitable quality education and promote lifelong learning opportunities for all, and more specifically in the fulfillment of goal 4.3 which consists of ensuring by the year 2030 the equal access of all men and women to quality technical, vocational and higher education, including university teaching, this, with the objective of verifying the contribution of HEIs to the achievement of said goal.

For this, quantitative analysis tools were applied to data obtained from the document Statistical Information Results of Higher Education Institutions 2016. Descriptive statistics were used on the time series of the period 2012-2016 to determine the trend with respect to the enrollment of students, disaggregating them according to sex, and also, the gender parity index (GPI) was applied on the data of that period.

Keywords: access to education, sustainable development objectives, higher education.

\section{Introducción}

En la historia reciente, la Organización de las Naciones Unidas se ha convertido en un foro trascendental respecto a las líneas de acción que deben impulsarse para lograr una mejora en aspectos clave en la vida de los seres humanos. Así, ya en septiembre del año 2002, líderes de 189 países se reunión en Nueva York para firmar la Declaración del Milenio, la cual se constituyó en un documento histórico en el que se comprometieron a alcanzar, antes de 2015, una serie de objetivos que son: 1) Erradicar la pobreza extrema y el hambre; 2) Lograr la enseñanza primaria universal; 3) Promover la igualdad entre los sexos y el empoderamiento de las mujeres; 4) Reducir la mortalidad de los niños menores de 5 años; 5) Mejorar la salud materna; 6) Combatir el VIH/SIDA, la malaria y otras enfermedades; 7) Garantizar la sostenibilidad del medio ambiente; y 8) Fomentar una alianza mundial para el desarrollo (Fondo ODS, s.f. a).
Los Objetivos del Milenio fueron revolucionarios porque ofrecieron un discurso común para alcanzar el acuerdo global, y además los ocho objetivos fueron realistas, fáciles de comunicar y contaron con un mecanismo claro para su cuantificación y seguimiento. El progreso alcanzado con los Objetivos del Milenio fue muy importante; sin embargo, el alcance de los logros fue desigual. Es por ello, que en septiembre del año 2015 los ODM fueron reemplazados por la nueva Agenda 2030 para el desarrollo Sostenible, la cual fue aprobada en la ciudad de Nueva York por 193 Estados Miembros de Naciones Unidas, enfocándose en la construcción de un mundo sostenible en el que se valoren la sostenibilidad del medio ambiente, la inclusión social y el desarrollo económico, abordando además las causas fundamentales de la pobreza y la necesidad universal de desarrollo que funcione para todas las personas. De esa manera, dicha agenda, se orienta a un conjunto de objetivos integrales enfocados en las personas, el planeta, 
la prosperidad, la paz y las alianzas (Fondo ODS, s.f. a; Fondo ODS, s.f. b; PNUD, s.f.)

En la agenda 2030, se plantearon un total de 17 objetivos desglosados en 169 metas, que requieren de alianzas entre actores tradicionales y no tradicionales, incluyendo Gobiernos, sociedad civil, sector empresarial y la Organización de las Naciones Unidas (Sostenibilidad para todos, s.f.).

Los 17 objetivos son: 1) Poner fin a la pobreza en todas sus formas en todo el mundo; 2) Poner fin al hambre, lograr la seguridad alimentaria y la mejora de la nutrición y promover la agricultura sostenible; 3) Garantizar una vida sana y promover el bienestar para todos en todas las edades; 4) Garantizar una educación inclusiva, equitativa y de calidad y promover oportunidades de aprendizaje durante toda la vida para todos; 5) Lograr la igualdad entre los géneros y empoderar a todas las mujeres y las niñas; 6) Garantizar la disponibilidad de agua y su gestión sostenible y el saneamiento para todos; 7) Garantizar el acceso a una energía asequible, segura, sostenible y moderna para todos; 8) Promover el crecimiento económico sostenido, inclusivo y sostenible, el empleo pleno y productivo y el trabajo decente para todos; 9) Construir infraestructuras resilientes, promover la industrialización inclusiva y sostenible, y fomentar la innovación; 10) Reducir la desigualdad en y entre los países; 11) Lograr que las ciudades y los asentamientos humanos sean inclusivos, seguros, resilientes y sostenibles; 12) Garantizar modalidades de consumo y producción sostenibles; 13) Adoptar medidas urgentes para combatir el cambio climático y sus efectos; 14) Conservar y utilizar en forma sostenible los océanos, los mares y los recursos marinos para el desarrollo sostenible; 15) Promover el uso sostenible de los ecosistemas terrestres, luchar contra la desertificación, detener e invertir la degradación de las tierras y frenar la pérdida de la diversidad biológica; 16) Promover sociedades pacíficas e inclusivas para el desarrollo sostenible, facilitar el acceso a la justicia para todos y crear instituciones eficaces, responsables e inclusivas a todos los niveles; y 17) Fortalecer los medios de ejecución y revitalizar la Alianza Mundial para el Desarrollo Sostenible (CEPAL, 2016).

En este estudio, nos enfocaremos en el objetivo 4 de garantizar una educación inclusiva, equitativa y de calidad y promover oportunidades de aprendizaje durante toda la vida para todos, y de manera específica en el cumplimiento de la meta 4.3 que consiste en asegurar al año 2030 el acceso igualitario de todos los hombres y las mujeres a una formación técnica, profesional y superior de calidad, incluida la enseñanza universitaria, ello, con el objetivo de verificar la contribución de las IES al logro de dicha meta.

\section{Justificación}

Respecto a los Objetivos de Desarrollo Sostenible, cabe mencionar que el Gobierno de E1 Salvador se encuentra actualmente trabajando en la elaboración de una Hoja de Ruta que se ha focalizado en 9 de los 17 Objetivos de Desarrollo Sostenible, los cuales están relacionados con la pobreza extrema, la salud, la educación, la igualdad de género, agua, acción climática, paz y justicia, y seguridad (Secretaría Técnica y de Planificación, 2018).

De dichos objetivos, se vuelve de vital importancia el vinculado con la educación, dado que la educación se considera como una llave, ya que esta permite el desarrollo y disfrute 
de diversos derechos individuales y colectivos, contribuyendo además al pleno desarrollo de las personas (Ronconi, 2018).

En ese sentido, el objetivo 4 de garantizar una educación inclusiva, equitativa y de calidad y promover oportunidades de aprendizaje durante toda la vida para todos, es prioritario para el Gobierno, por lo que resulta de alta importancia realizar estudios que nos permitan brindar un panorama sobre los avances en el logro de dicho objetivo, de forma que puedan focalizarse esfuerzos en aquellos aspectos importantes en lo que aún se puede avanzar.

Es por ello relevante conocer el aporte de las instituciones de educación superior (IES) al logro del objetivo $4 \mathrm{y}$ al alcance de sus metas, por lo que a través del presente estudio se verificará la contribución de las IES al alcance de la meta 4.3 que consiste en asegurar al año 2030 el acceso igualitario de todos los hombres y las mujeres a una formación técnica, profesional y superior de calidad, incluida la enseñanza universitaria.

\section{Marco conceptual}

Para el desarrollo del estudio, es importante comprender algunos conceptos clave que se encuentran vinculados con el objeto de la investigación, como lo son desarrollo sostenible, educación y objetivos del desarrollo sostenible.

\section{Educación superior e instituciones de educación superior}

La expresión de educación superior, se refiere al tercer nivel del sistema educativo, que se articula generalmente en dos niveles, pregrado y posgrado, y se desarrolla en instituciones de educación superior (IES), término que engloba diferentes tipos de organizaciones o centros de estudios (Díaz Buitrago, Alvarino Bettín, \& Carrascal Torres, 2011).

Por su parte, para la UNESCO (2008), se entiende por educación superior "todo tipo de estudios, de formación o de formación para la investigación en el nivel postsecundario, impartidos por una universidad u otros establecimientos de enseñanza que estén acreditados por las autoridades competentes del Estado como centros de enseñanza superior" (p. 19).

Para la legislación salvadoreña, específicamente en la Ley General de Educación (Asamblea Legislativa de El Salvador, 2004), en su Art. 4, se entenderá por educación superior "todo esfuerzo sistemático de formación posterior a la enseñanza media y comprende: La Educación Tecnológica y la Educación Universitaria” (p. 2).

Además, en dicha ley, en su Art. 22, se establecen las organizaciones facultadas para impartir la educación superior, las cuales se definen como instituciones de educación superior y son los institutos tecnológicos, los institutos especializados de nivel superior, y las universidades (Asamblea Legislativa de El Salvador, 2004).

En ese mismo Art. 22, se define que los institutos tecnológicos son aquellos dedicados a la formación de técnicos y tecnólogos en las distintas especialidades científicas, artísticas y humanísticas. Por su parte, los institutos especializados de nivel superior, son los dedicados a formar profesionales en un área de las ciencias, la técnica o el arte, y finalmente, las 
universidades, son las dedicadas a la formación académica en carreras con estudios de carácter multidisciplinario en las ciencias, artes y técnicas (Asamblea Legislativa de E1 Salvador, 2004).

\section{Acceso igualitario entre hombres y mujeres a la educación}

Para poder comprender lo que se entiende por acceso igualitario de hombres una formación técnica, profesional y superior de calidad, es necesario comprender la significancia del concepto de igualdad. Así pues, cuando hablamos de igualdad como sustantivo, puede hacerse referencia a la relación entre dos cosas semejantes, sin embargo, cuando trasladamos este concepto al plano de la igualdad entre hombres y mujeres, éste adquiere una significancia mayor, pues implica un trato idéntico o diferenciado entre hombres y mujeres que resulta en una total ausencia de cualquier forma de discriminación contra las mujeres por ser mujeres (Facio, 2009)

Así pues, en el campo de lo social, y especialmente desde el punto de vista de los derechos humanos, la igualdad es vista como una situación o contexto donde las personas tienen los mismos derechos y las mismas oportunidades en un determinado aspecto o a nivel general, y al hablar de igualdad de sexo o de género, se hace referencia además a la eliminación del estándar masculino en el acceso a las oportunidades existentes para que puedan repartirse de manera justa entre hombres y mujeres (Facio, 2016)

$\mathrm{Al}$ aplicar entonces, el concepto de igualdad entre hombres y mujeres en relación al acceso igualitario, se hace referencia a que deben brindarse las mismas oportunidades tanto a hombres como a mujeres para poder ingresar al sistema educativo, lo cual permitirá en un mediano plazo romper con la concepción de la educación como un medio para la reproducción de los esquemas sociales de desigualdad y la perpetuación de las identidades de género socialmente determinadas (Castillo Sánchez \& Gamboa Araya, 2013). La igualdad de oportunidades desde una perspectiva socioeducativa estaría contribuyendo de esa manera no solo a la generación de conocimiento, sino además al desarrollo de las competencias y habilidades de inserción laboral y social que favorezcan la plena igualdad entre sexos (García Perales, 2012).

\section{Metodología}

La investigación ha sido desarrollada con un alcance de tipo exploratorio, aplicando herramientas de análisis cuantitativo a fuentes secundarias generadas por el Ministerio de Educación de El Salvador, específicamente a datos obtenidos del documento Resultados de la Información Estadística de Instituciones de Educación Superior 2016 (MINED, 2017).

Para el análisis de los datos contenidos en dicho documento, se aplicó estadística descriptiva sobre las series de tiempo del período 20122016 para determinar la tendencia respecto a la inscripción de estudiantes en razón del sexo en las instituciones de educación superior de E1 Salvador. Además, se aplicó el índice de paridad entre los géneros (IPG) (UNESCO, 2009) sobre los datos del período 2012-2016 con lo cual, se verificó la contribución de las instituciones de educación superior al alcance de la meta 4.3 que consiste en asegurar al año 2030 el acceso igualitario de todos los hombres y las mujeres a 
una formación técnica, profesional y superior de calidad, incluida la enseñanza universitaria.

\section{Resultados}

E1 Ministerio de Educación, a partir de la implementación de la Ley de Educación Superior de finales de 1995 y las reformas efectuadas en noviembre de 2004 y julio de 2008, ha venido desarrollado de forma permanente y participativa el proceso de Información Estadística de Educación Superior, lo cual ha permitido contar con información fiable y útil para el análisis de la situación de la educación superior en E1 Salvador (MINED, 2017).

Dicha información es utilizada en este estudio para constatar la contribución de las instituciones de educación superior de El Salvador al aseguramiento del acceso igualitario de hombres y mujeres a una formación técnica, profesional y superior de calidad, a partir del análisis estadístico. Asimismo, se utilizan dichos datos para calcular el índice de paridad entre los géneros (IPG), el cual, forma parte de los indicadores de la educación generados en el año 2009 por la UNESCO y que tiene por finalidad medir el avance hacia la paridad entre los géneros en términos de la participación en la educación y/o las oportunidades de aprendizaje abiertas a la mujer relativas a las disponibles al hombre (UNESCO, 2009).

Así pues, el IPG es entonces una relación entre el valor correspondiente al sexo femenino $y$ el correspondiente al sexo masculino para un indicador dado, y resulta de dividir el valor femenino de un indicador determinado por el valor masculino (UNESCO, 2009).
Al verificar los datos agregados de la matrícula de estudiantes en las IES de El Salvador para el período 2012-2016 que refleja el documento Resultados de la Información Estadistica de Instituciones de Educación Superior 2016, se puede observar, como muestra la Figura n. ${ }^{\circ}$, que la matrícula tuvo un incremento global de 11,095 estudiantes, lo que indica un crecimiento porcentual del $6.53 \%$ en el período mencionado. En relación al crecimiento anual, se puede observar que el año con mayor crecimiento fue el 2013 con 6,203 estudiantes, y el de menor crecimiento en relación al año anterior fue el 2014 con 263 estudiantes.

Al desagregar los estudiantes por sexo, se observa que el año con un mayor incremento respecto a estudiantes tanto de sexo masculino como de sexo femenino fue de la misma forma el año 2013 con un crecimiento de 2,749 estudiantes del sexo masculino y de 3,454 del sexo femenino.

Ver Figura n. ${ }^{\circ} 1$.

Si se verifica la variación de estudiantes del año 2015, año en que se aprobó la Agenda 2030, hacia el año 2016, se observa que el incremento en la matrícula total de estudiantes en las instituciones de educación superior fue únicamente de 1,559 estudiantes, lo que representa un crecimiento porcentual de tan solo $0.87 \%$. De esos estudiantes, 417 son de sexo masculino y 1,142 son del sexo femenino, lo que representa un crecimiento porcentual del $0.50 \%$ en el sexo masculino y de $1.19 \%$ en el sexo femenino. Sin embargo, al verificar el índice de paridad entre los géneros (IPG) en la Figura n. ${ }^{\circ} 2$, se observa que, si bien para el período analizado en todos los años ha sido favorable para el sexo femenino, 


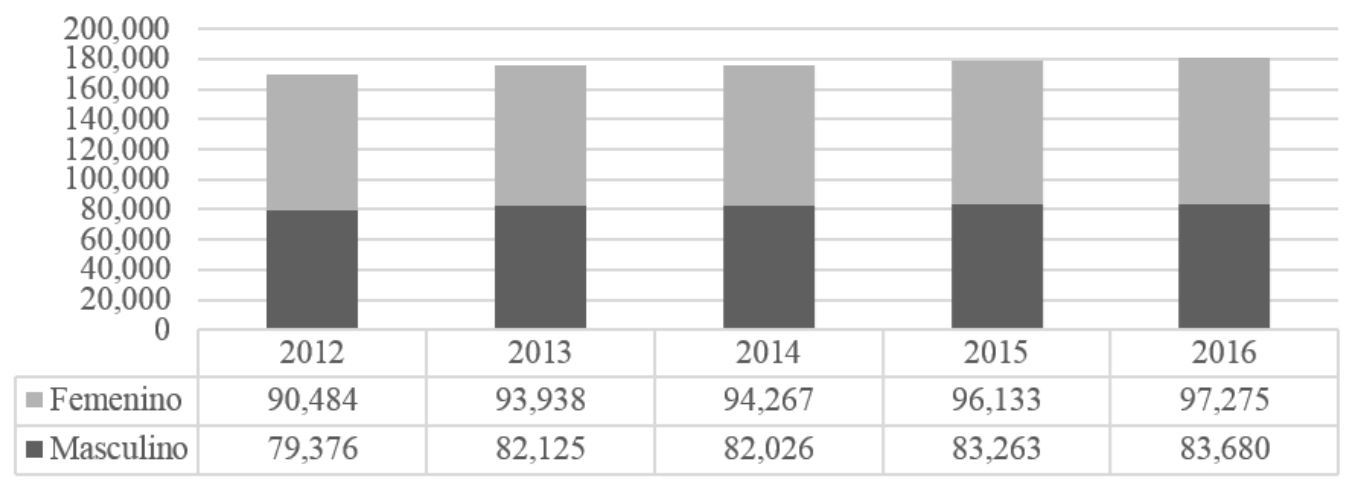

Figura $n .^{\circ} 1$. Matrícula de instituciones de educación superior 2012-2016. Fuente: elaboración propia a partir de datos del MINED (2017).

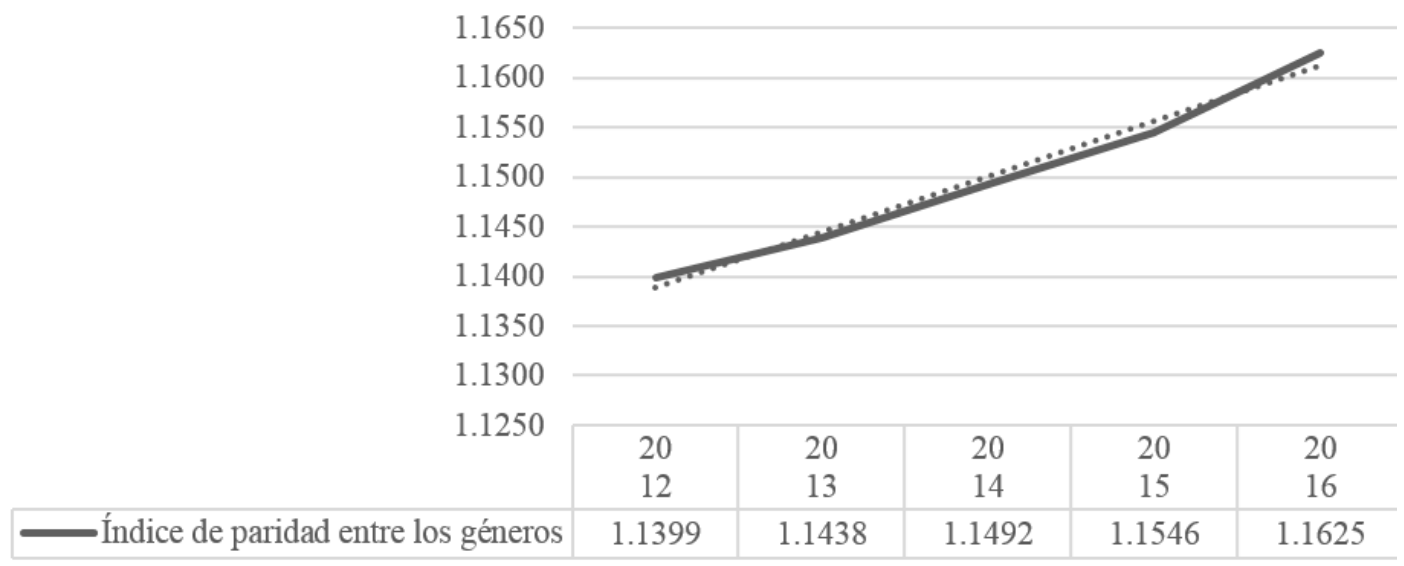

Figura . $^{\circ}$ 2. Índice de paridad de los géneros (IPG) en IES 2012-2016. Fuente: elaboración propia a partir de datos del MINED (2017).

es para este año 2016 en el cual se constata una relación mayor de estudiantes de sexo femenino respecto a los estudiantes de sexo masculino, con un IPG de 1.1625, lo cual representa un logro respecto a la meta 4 la meta 4.3 que consiste en asegurar al año $2030 \mathrm{el}$ acceso igualitario de todos los hombres y las mujeres a una formación técnica, profesional y superior de calidad, incluida la enseñanza universitaria, ya que dicha meta se establece en razón de que históricamente ha habido un menor acceso de mujeres a la educación, por lo cual se valora positivamente un incremento en el acceso para las mujeres 
Si bien, estas cifras agregadas de las instituciones de educación superior nos presentan un panorama positivo respecto al acceso de las mujeres a una formación técnica, profesional y superior de calidad, resulta interesante verificar las tendencias en cada tipo de institución de educación superior, siendo estas clasificadas como universidades, institutos especializados e institutos tecnológicos.

Para el caso de las universidades, durante el período 2012-2016, se puede observar, como muestra la Figura n. ${ }^{\circ}$, que la matrícula tuvo un incremento global de 11,964 estudiantes, lo cual indica un crecimiento porcentual del $7.67 \%$ en dicho período. Respecto al crecimiento anual, se observa que el año con mayor crecimiento fue el 2013 con 6,524 estudiantes, y el de menor crecimiento fue el 2014 con 1,029 estudiantes.

Al desagregar los estudiantes por sexo, se observa que el año con un mayor incremento respecto a estudiantes de sexo masculino fue de la misma forma el año 2013 con un crecimiento de 3,020 estudiantes, y asimismo, lo fue para el sexo femenino con un crecimiento de 3,504 estudiantes.

Si se verifica la variación de estudiantes del año 2015, año en que se aprobó la Agenda 2030, hacia el año 2016, se observa que el incremento en la matrícula total de estudiantes en las universidades fue únicamente de 1,392 estudiantes, lo que representa un crecimiento porcentual de tan solo $0.84 \%$. De esos estudiantes, 196 son de sexo masculino y 1,196 son del sexo femenino, lo que representa un crecimiento porcentual del 0.26 $\%$ en el sexo masculino y de $1.33 \%$ en el sexo femenino. Sin embargo, al verificar el índice de paridad entre los géneros (IPG), se observa como muestra la Figura n. ${ }^{\circ}$ 4, que, si bien para el período analizado en todos los años ha sido favorable para el sexo femenino, es para este año 2016 en el cual se constata una relación mayor de estudiantes de sexo femenino respecto a los estudiantes de sexo masculino, lo cual llega a representar un logro mayor respecto a la meta 4 la meta 4.3 que consiste en asegurar al año 2030 el acceso igualitario de todos los hombres y las mujeres a una formación técnica, profesional y superior de calidad, incluida la enseñanza universitaria.

Respecto a los institutos especializados, se puede observar, por el contrario, que para el período 2012-2016, como muestra la Figura n. ${ }^{\circ}$ 5, la matricula tuvo una disminución global de 360 estudiantes, lo que indica un decrecimiento porcentual del $3.08 \%$ en dicho período. Respecto al crecimiento anual, se observa que el año con mayor crecimiento fue el 2016 con 265 estudiantes, mientras que el mayor decrecimiento se presentó en el año 2014 con 523 estudiantes menos con respecto al año anterior.

Al desagregar los estudiantes por sexo, se observa que el año con un mayor incremento respecto a estudiantes de sexo masculino fue el año 2016 con un crecimiento de 249 estudiantes, mientras que para estudiantes del sexo femenino lo fue el año 2015 con un crecimiento de 59 estudiantes.

Si se verifica la variación de estudiantes del año 2015, año en que se aprobó la Agenda 2030, hacia el año 2016, se observa que es el año con mayor incremento de estudiantes en el período, con un total de 265 estudiantes, lo que representa un crecimiento porcentual de $2.40 \%$. De esos estudiantes, 249 son de sexo masculino y 16 son del sexo femenino, lo que representa 


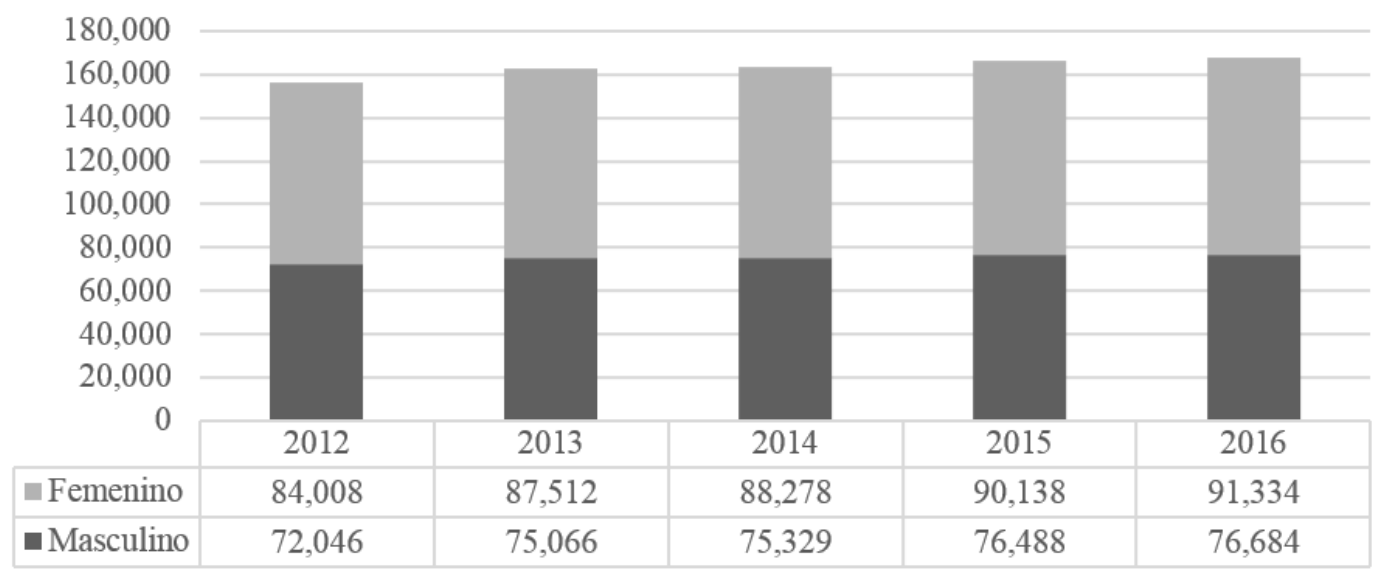

Figura $n .^{\circ} 3$. Matrícula de universidades 2012-2016. Fuente: elaboración propia a partir de datos del MINED (2017).

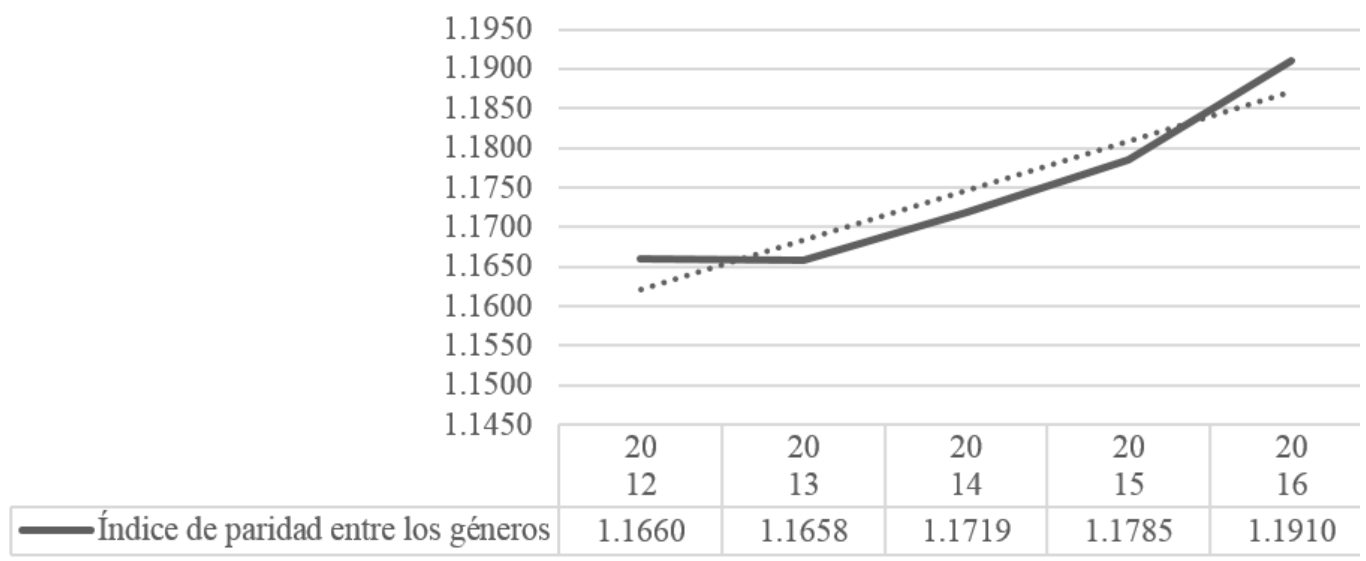

Figura $n .^{\circ}$ 4. Índice de paridad entre los géneros (IPG) en universidades 2012-2016.

Fuente: elaboración propia a partir de datos del MINED (2017).

un crecimiento porcentual del $4.17 \%$ en el sexo masculino y de $0.32 \%$ en el sexo femenino. Sin embargo, al verificar el índice de paridad entre los géneros (IPG) para el año 2016, en la Figura n. ${ }^{\circ}$, se observa que es el año en el cual existe una mayor relación desfavorable del sexo femenino respecto al masculino. Cabe mencionar que los datos del índice de paridad de los géneros reflejan una tendencia desfavorable para todo el período 2012-2016, con lo cual se constata que en relación a los institutos especializados existe aún camino que recorrer para aumentar 


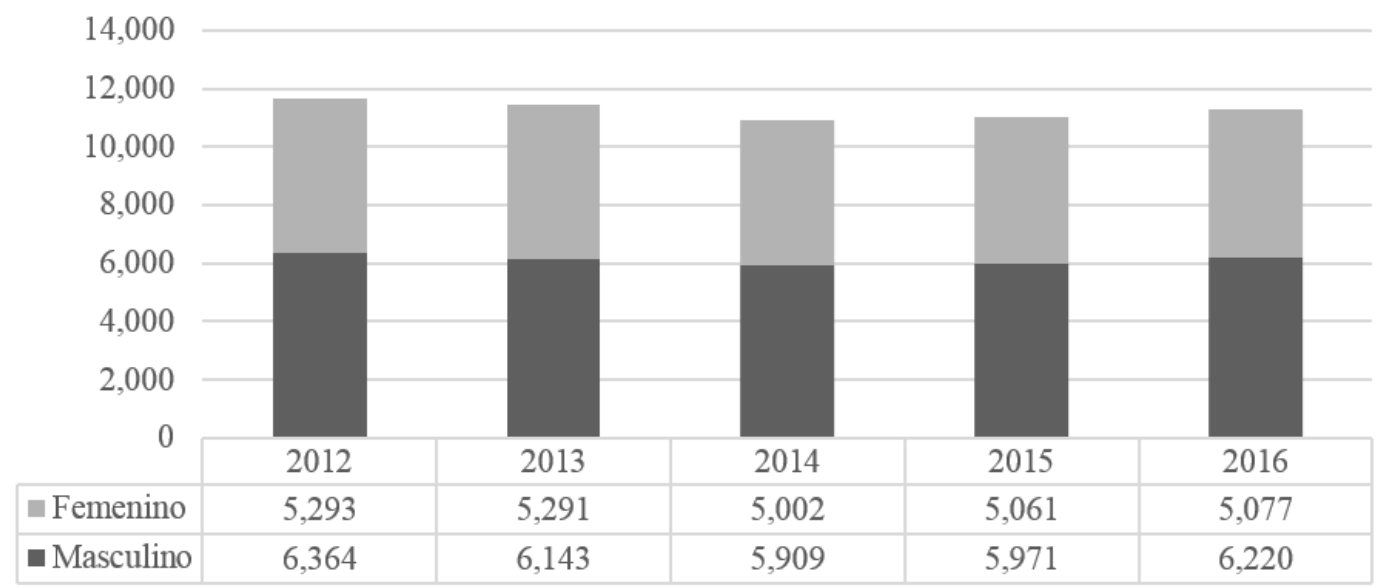

Figura $n .^{\circ}$ 5. Matrícula de institutos especializados 2012-2016. Fuente: elaboración propia a partir de datos del MINED (2017).

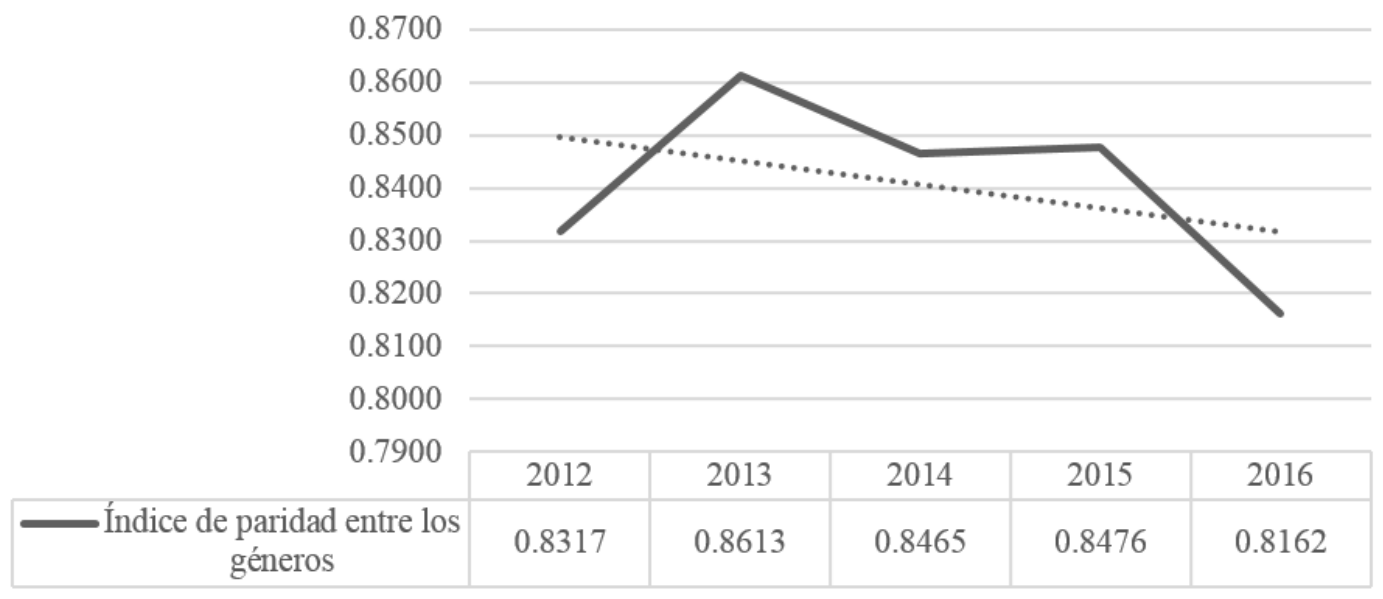

Figura $n .^{\circ}$ 6. Índice de paridad entre los géneros (IPG) en institutos especializados 2012-2016. Fuente: elaboración propia a partir de datos del MINED (2017).

la participación de mujeres y para contribuir en mejor manera al logro de la meta 4.3 de los ODS.

Por otra parte, respecto a los institutos tecnológicos, se puede observar, que al igual que los institutos especializados, para el período 2012-2016, la matrícula también tuvo una disminución global, que fue de 590 estudiantes, lo que indica un decrecimiento porcentual del $23.69 \%$ en dicho período. Respecto al período 
se observa que en todos los años ha habido decrecimiento en relación al año anterior, y se observa que el año con menor decrecimiento fue el 2015 con 37 estudiantes menos, mientras que el mayor decrecimiento se presentó en el año 2016 con 98 estudiantes menos con respecto al año anterior.

Al desagregar los estudiantes por sexo, se observa, como muestra la Figura n. ${ }^{\circ}$, que el año con un mayor incremento respecto a estudiantes de sexo masculino fue el año 2015 con un crecimiento de 16 estudiantes, mientras que para estudiantes del sexo femenino en todos los años hubo decrecimiento en relación al año anterior, y el año con menor decrecimiento fue el año 2013 con una disminución de 48 estudiantes respecto al año anterior.

Si se verifica la variación de estudiantes del año 2015, se observa, como ya se mencionó anteriormente, que el año 2016 es el que presenta mayor decrecimiento de estudiantes, con un total de 98 estudiantes menos, lo que representa un decrecimiento porcentual de 5.64 $\%$. De esos estudiantes, 28 son de sexo masculino y 70 son del sexo femenino, lo que representa un decrecimiento porcentual del $3.48 \%$ en el sexo masculino y de $7.49 \%$ en el sexo femenino respecto al año anterior. Al verificar el índice de paridad entre los géneros (IPG) para el año 2016, como muestra Figura n. ${ }^{\circ}$, se observa que es de 1.1134 lo cual es favorable para el sexo femenino; sin embargo, es el año del período 2012-2016 en el que el índice es menor. Lo que cabe destacar es que, en relación a los institutos tecnológicos, el índice de paridad entre los géneros ha sido favorable para el sexo femenino en todo el período analizado, con lo cual se constata que se está contribuyendo al logro de la meta 4.3 de los ODS.

Por otra parte, respecto a los institutos tecnológicos, se puede observar, que al igual que los institutos especializados, para el período 2012-2016, la matrícula también tuvo una disminución global, que fue de 590 estudiantes, lo que indica un decrecimiento porcentual del $23.69 \%$ en dicho período. Respecto al período se observa que en todos los años ha habido decrecimiento en relación al año anterior, y se observa que el año con menor decrecimiento fue el 2015 con 37 estudiantes menos, mientras que el mayor decrecimiento se presentó en el año 2016 con 98 estudiantes menos con respecto al año anterior.

Al desagregar los estudiantes por sexo, se observa, como muestra la Figura n. ${ }^{\circ}$ 7, que el año con un mayor incremento respecto a estudiantes de sexo masculino fue el año 2015 con un crecimiento de 16 estudiantes, mientras que para estudiantes del sexo femenino en todos los años hubo decrecimiento en relación al año anterior, y el año con menor decrecimiento fue el año 2013 con una disminución de 48 estudiantes respecto al año anterior.

Si se verifica la variación de estudiantes del año 2015, se observa, como ya se mencionó anteriormente, que el año 2016 es el que presenta mayor decrecimiento de estudiantes, con un total de 98 estudiantes menos, lo que representa un decrecimiento porcentual de 5.64 $\%$. De esos estudiantes, 28 son de sexo masculino y 70 son del sexo femenino, lo que representa un decrecimiento porcentual del $3.48 \%$ en el sexo masculino y de $7.49 \%$ en el sexo femenino respecto al año anterior. Al verificar el índice de paridad entre los géneros (IPG) para el año 


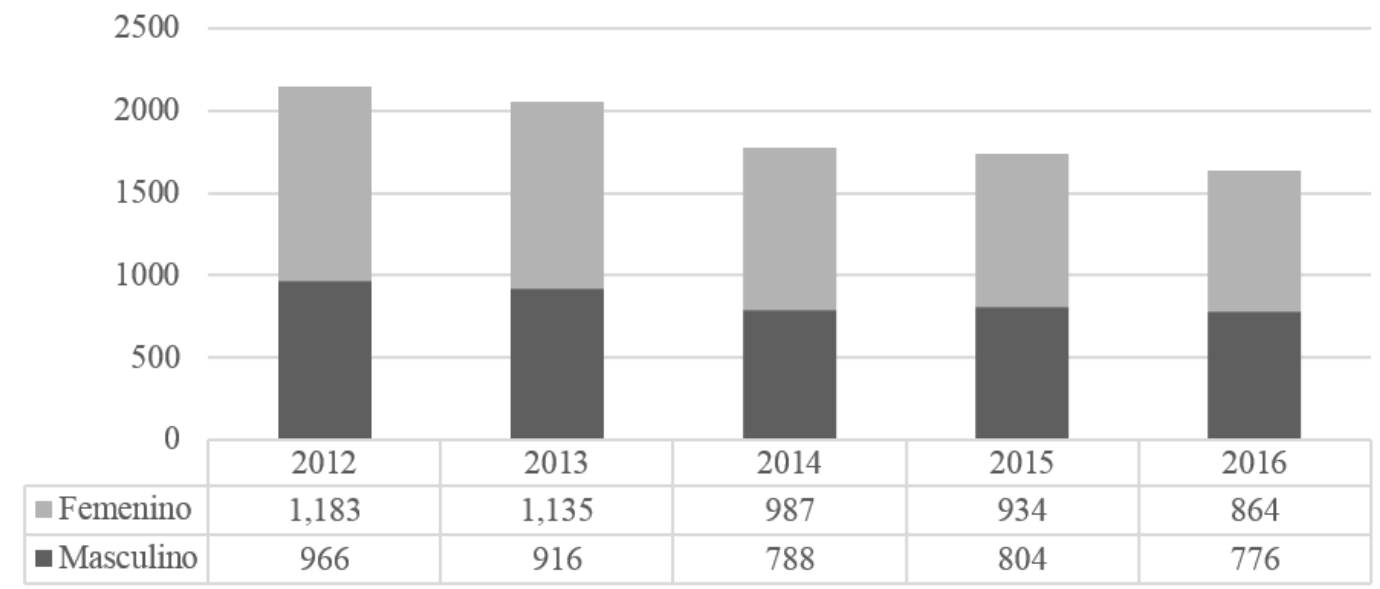

Figura $n .^{\circ}$ 7. Matrícula de institutos tecnológicos 2012-2016. Fuente: elaboración propia a partir de datos del MINED (2017).

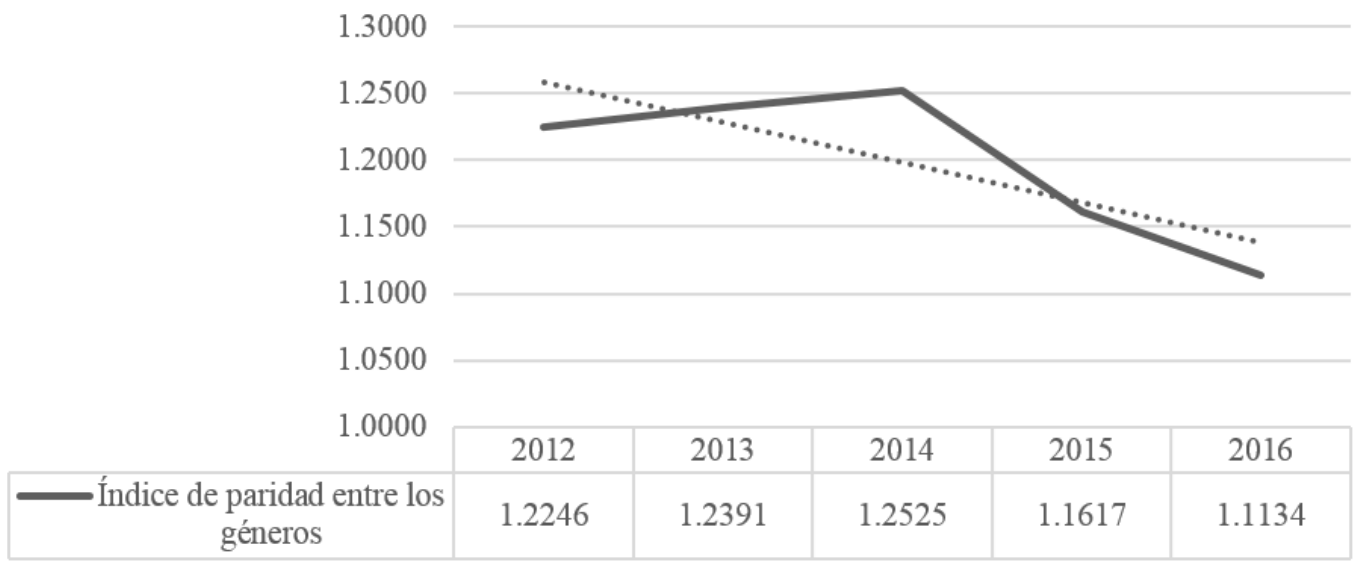

Figura $n .^{\circ} 8$. Índice de paridad entre los géneros (IPG) en institutos tecnológicos 2012-2016. Fuente: elaboración propia a partir de datos del MINED (2017).

2016, como muestra Figura n. ${ }^{\circ}$, se observa que es de 1.1134 lo cual es favorable para el sexo femenino; sin embargo, es el año del período 2012-2016 en el que el índice es menor. Lo que cabe destacar es que, en relación a los institutos tecnológicos, el índice de paridad entre los géneros ha sido favorable para el sexo femenino en todo el período analizado, con lo cual se constata que se está contribuyendo al logro de la meta 4.3 de los ODS. 
Habiendo analizado entonces los datos para cada uno de los tipos de instituciones de educación superior, se puede observar que son las universidades las que han presentado un mayor incremento en su matrícula durante el período 2012-2016 tanto de estudiantes del sexo masculino como del sexo femenino, y asimismo, reflejan un IPG constantemente favorable para el sexo femenino en relación con el masculino, con una tendencia alcista.

Otro dato interesante es el resultado en los institutos tecnológicos, en los cuales, si bien se ha venido dando durante el período analizado una disminución en el total de estudiantes matriculados, respecto al acceso igualitario de todos los hombres y las mujeres a una formación técnica, profesional y superior de calidad, se puede observar que el IPG a pesar de mostrar una tendencia bajista, aún se mantiene en una relación favorable para el sexo femenino.

Finalmente, por su parte, el caso de los institutos especializados presenta un panorama preocupante según el período analizado, dado que su matrícula de estudiantes presenta una tendencia bajista, así como también un IPG constantemente desfavorable para el sexo femenino en relación al masculino y que se presenta también con una tendencia bajista.

Ahora bien, agregado a esto, resulta también interesante verificar qué tipo de carreras son la que estudian las mujeres, si existen carreras feminizadas y si las mujeres se están graduando de carreras modelo STEAM (ciencia, tecnología, ingeniería y matemáticas, por sus siglas en inglés).
Para efectuar dicho análisis, es importante comenzar señalando que el Ministerio de Educación ha definido diez áreas de formación profesional con el objeto de presentar grupos de carreras con similares características, siendo éstas las siguientes: i) Arte y Arquitectura; ii) Economía, Administración y Comercio;iii) Salud;iv) Ciencias; v) Agropecuaria y Medio Ambiente; vi) Derecho; vii) Humanidades; viii) Tecnología; ix) Educación y x) Ciencias Sociales (MINED, 2017).

Por otra parte, se debe señalar que de estas áreas, las de Salud, Educación y Ciencias Sociales han sido tradicionalmente reconocidas como femeninas (Ruiz Fernández, 2014), y para el caso de El Salvador, encontramos validez de dicho supuesto, ya que, de acuerdo a Duriez y Zamora (2016) la matrícula femenina en los últimos años en El Salvador ha predominado en las áreas de Economía, Administración y Comercio; Salud y Educación, mientras que la matrícula masculina predominó en Tecnología y Economía, Administración y Comercio, y asimismo, señalan que las mujeres en su mayoría no están accediendo a carreras de las Ciencias Naturales, Ciencias Agropecuarias, Arte y Tecnología de forma masiva.

Lo anterior es verificable con los datos del MINED (2017), tal como se muestra en la Figura n. ${ }^{\circ}$, en la cual se observa que para el período 2012 - 2016, las áreas de formación en las que más se inscriben mujeres son Economía; Administración y Comercio; Salud y Educación; mientras en las que menos se inscriben son Ciencias, Agropecuaria y Medio Ambiente, Arte y Arquitectura. Mientras que, por otra parte, en el caso de los hombres, como se muestra en la Figura n. ${ }^{\circ}$ 10, efectivamente se observa que para 


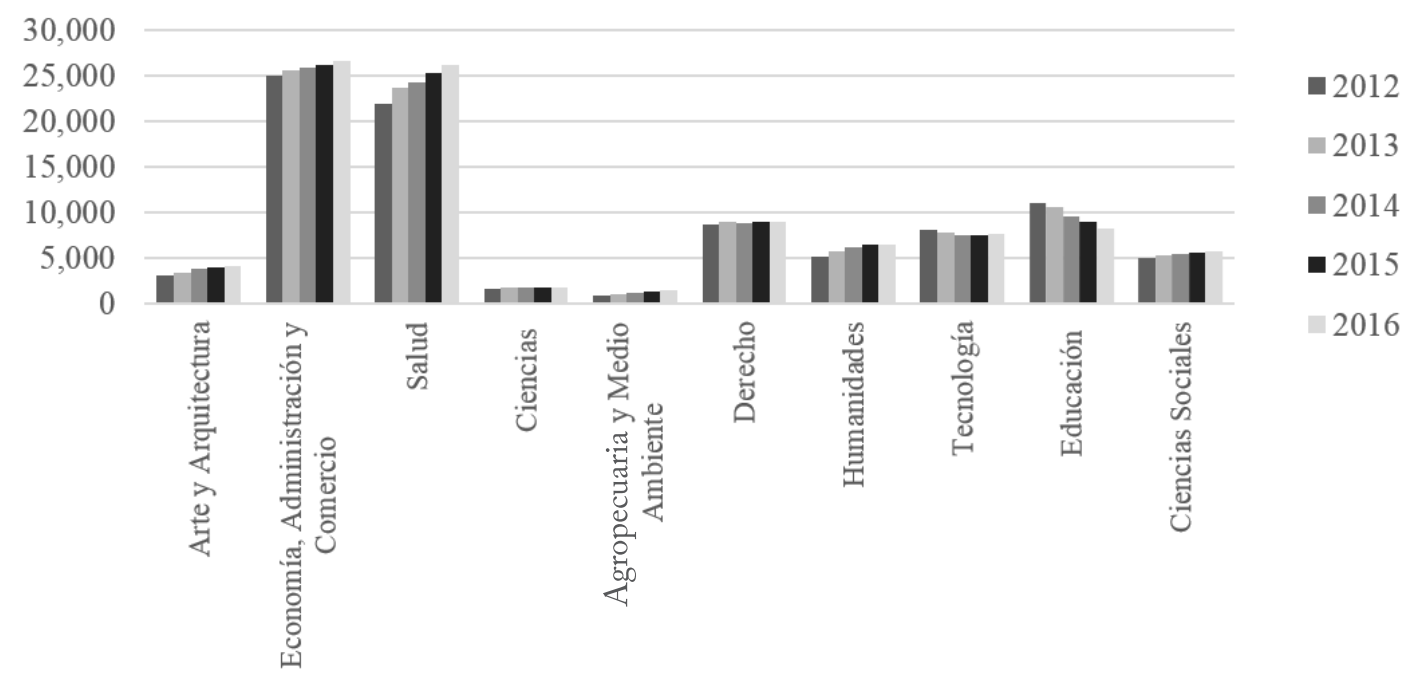

Figura $n .^{\circ}$ 9. Matrícula de mujeres por área de formación 2012-2016. Fuente: elaboración propia a partir de datos del MINED (2017).

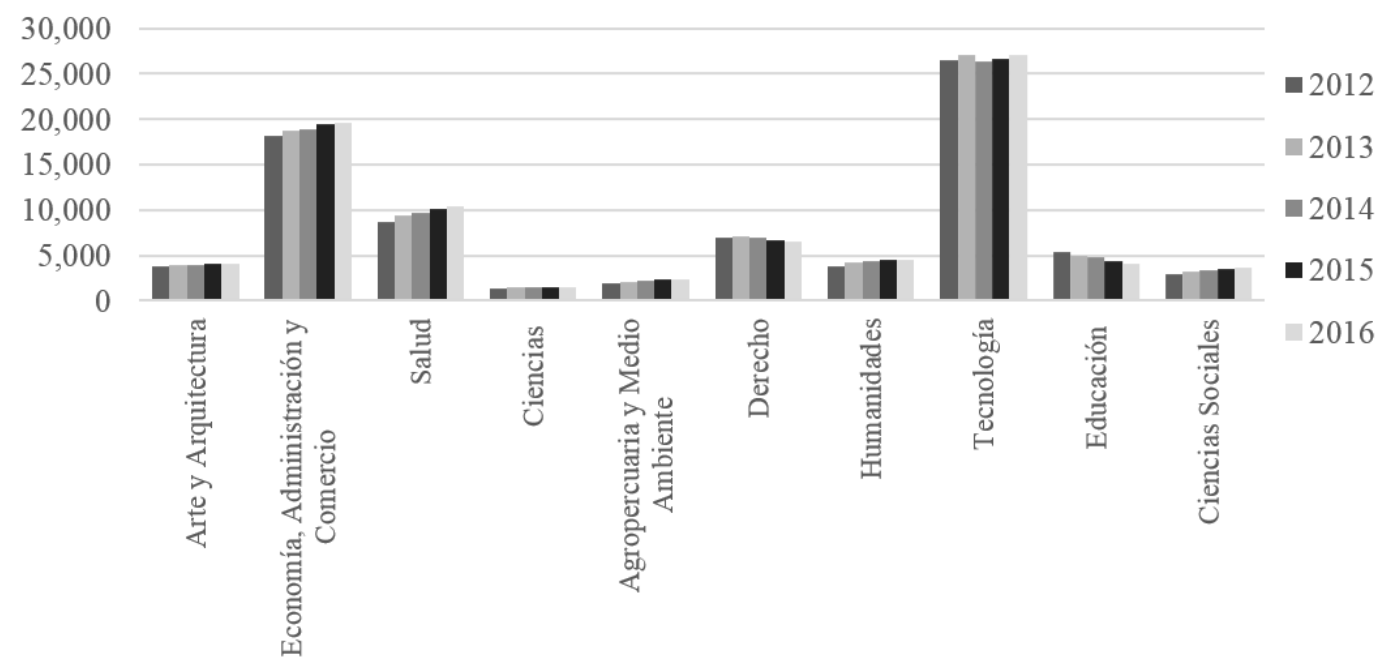

Figura $n .^{\circ}$ 10. Matrícula de hombres por área de formación 2012-2016. Fuente: elaboración propia a partir de datos del MINED (2017).

el período 2012 - 2016, las áreas de formación en las que más se inscriben son Tecnología y Economía, Administración y Comercio.
Por otra parte, cuando verificamos la cantidad de graduados por año, se puede observar, como muestra la Figura n. ${ }^{\circ} 11$, que para el 
período 2012 - 2016 se ha graduado un mayor número de mujeres que de hombres, y como muestra la Figura n. ${ }^{\circ}$ 12, el mayor número de mujeres se gradúa en las áreas de formación ya anteriormente identificadas como feminizadas y que son Economía, Administración y Comercio; Salud y Educación, mientras que el mayor número de hombres se gradúa en Tecnología y Economía, Administración y Comercio. En ese sentido, dicha información revela que las carreras STEM siguen siendo principalmente masculinizadas, especialmente respecto a las carreras enfocadas en tecnología e ingeniería, siendo aún muy limitado el número de mujeres que se gradúa de dichas carreras.

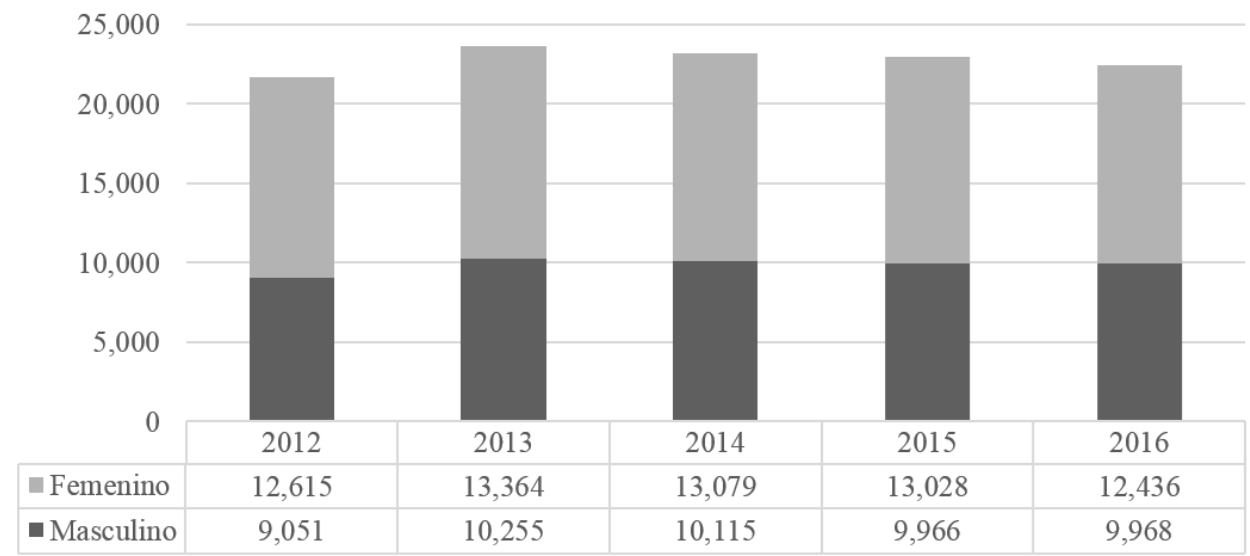

Figura n. ${ }^{\circ}$ 11. Graduados de educación superior desagregados por género 2012-2016.

Fuente: elaboración propia a partir de datos del MINED (2017).

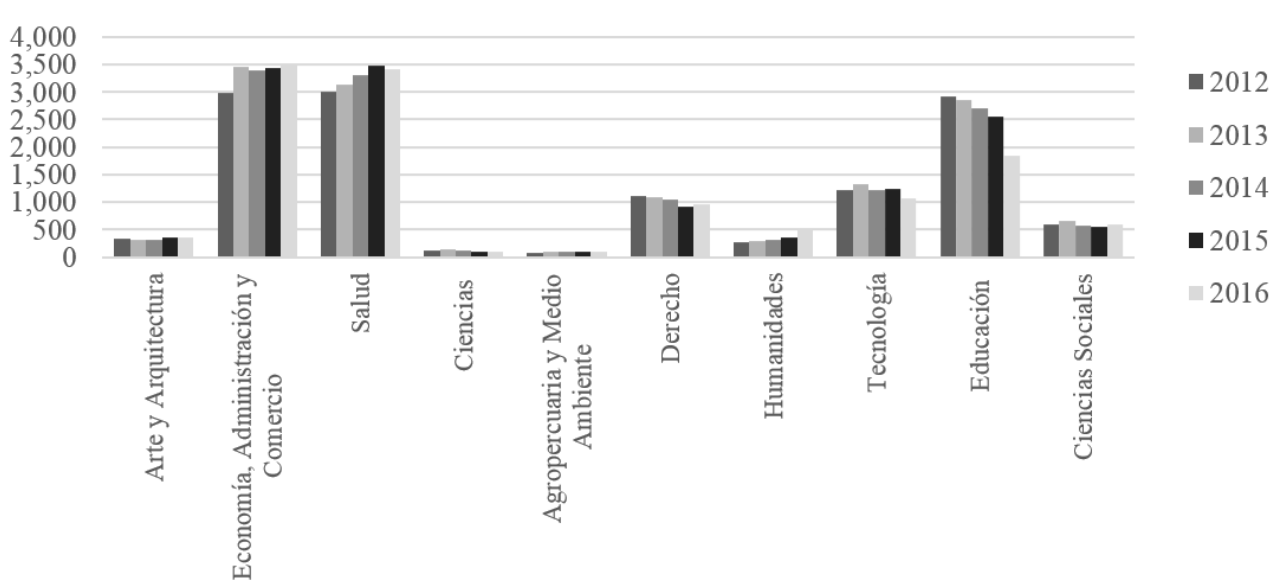

Figura $n .^{\circ}$ 12. Mujeres graduadas de educación superior por área de formación 2012-2016. Fuente: elaboración propia a partir de datos del MINED (2017). 


\section{Conclusiones}

Si bien, en análisis de datos nos ha permitido constatar una contribución de las instituciones de educación de El Salvador al logro de la meta 4.3 el objetivo 4 de los ODS, se ha observado que son las universidades las que contribuyen en mayor medida, mostrándose efectivamente en dichas instituciones un alza en el IPG del año 2016 en relación al año 2015, lo cual, podría atribuirse a la adopción de la Agenda 2030 para el Desarrollo Sostenible. Sin embargo, dado el alcance exploratorio del estudio, queda abierta la posibilidad de profundizar acerca de ello, verificando si efectivamente las universidades han impulsado el aseguramiento del acceso igualitario de hombres y mujeres a una formación técnica, profesional y superior de calidad a través políticas, acciones o medidas concretas para tal fin.

Por otra parte, se ha podido observar que, tanto en los institutos especializados como los institutos tecnológicos, presentan una tendencia bajista en su matrícula de estudiantes durante el período analizado, ello constituye un llamado de atención dado que en su mayoría son instituciones públicas financiadas a su vez con fondos públicos, por lo cual cabría verificar la oferta académica en este tipo de instituciones de educación superior, así como también el perfil de los estudiantes que optan por este tipo de formación, de forma que pueda haber una mayor vinculación entre la oferta y la demanda educativa para incentivar el acceso al tipo de educación que se ofrece en esas instituciones.

Además, el IPG tanto en institutos especializados como en los tecnológicos muestra una tendencia bajista, lo que indica que las mujeres están reduciendo su acceso a la educación técnica, lo cual no contribuye a la meta 4.3 que contempla el acceso a una educación técnica de calidad. En ese sentido, los datos reflejan una necesidad de impulsar políticas, medidas o acciones que permitan incentivar la formación tecnológica de las mujeres, para que puedan acceder de mejor manera a un mercado laboral que demanda cada vez más este tipo de formación.

Finalmente, si bien los datos analizados dan un panorama en general favorable respecto al acceso igualitario de hombres y mujeres a una formación técnica, profesional y superior de calidad, para poder contribuir en mayor medida al logro de la meta 4.3 y del objetivo 4 , se observa que, tanto en el ingreso como en la graduación, sigue existiendo una feminización de áreas de formación, especialmente en Economía, Administración y Comercio; Salud y Educación, mientras que en el áreas del modelo STEM, especialmente en la de Tecnología, sigue existiendo una masculinización, por lo que es necesario impulsar acciones, tanto desde el sector público como desde el sector privado, que tiendan a generar un cambio en los imaginarios colectivos y en la cultura, como por ejemplo en la preferencia de invertir en la educación de los varones y en la división sexista del trabajo. Además, las instituciones de educación superior deben considerar las necesidades particulares de las mujeres de forma que su proceso de formación sea compatible tanto con su vida laboral como familiar, y les permita además mayores niveles de participación en el desarrollo social y económico y en los espacios políticos, para lo cual es importante la transversalización de la cultura de género a través de políticas institucionales.

En la medida en que tanto en la sociedad como en las IES se fortalezca la conciencia sobre la 
igualdad de género y se impulsen medidas para promoverla, en esa medida se logrará un mayor avance no solamente en el cumplimiento de la meta 4.3 del objetivo 4 de los ODS, sino también en el mejoramiento de la calidad de vida de los habitantes logrando de esa manera un verdadero desarrollo sostenible centrado en personas, el planeta, la prosperidad, la paz y las alianzas.

\section{Bibliografía}

Asamblea Legislativa de E1 Salvador. (2004). Ley General de Eduación Superior. San Salvador.

Castillo Sánchez, M., \& Gamboa Araya, R. (2013). La vinculación de la educación y género. Actualidades Investigativas en Educación, 1-16.

CEPAL. (2016). Agenda 2030 y los Objetivos de Desarrollo Sostenible. Una oportunidad para América Latina y el Caribe. Santiago: Naciones Unidas.

Díaz Buitrago,E.,Alvarino Bettín, G., \& Carrascal Torres, N. (2011). Enfoques de aprendizaje y niveles de comprensión. El aprendizaje universitario en ambientes tecnológicos. Montería: Fondo Editorial Universidad de Córdova.

Duriez González, M., \& Zamora Arrechavala, B. (2016). Educación superior en Iberoamérica. Informe 2016. Información Nacional: El Salvador. CINDA.

Facio, A. (2009). Igualdad en la CEDAW; 30 años de desarrollo de un derecho clave para las mujeres. CEDAW/C/39/D/7/2005.

Facio, A. (2016). La responsabilidad estatal frente al derecho humano a la igualdad. México
D. F.: Comisión de Derechos Humanos del Distrito Federal.

Fondo ODS. (s.f. a). Fondo para los Objetivos de Desarrollo Sostenible. Obtenido de http://www. sdgfund.org/es/de-los-odm-los-ods

Fondo ODS. (s.f. b). Fondo para los Objetivos de Desarrollo Sostenible. Obtenido de Objetivos de Desarrollo Sostenible: http://www.sdgfund.org/ es/objetivos-de-desarrollo-sostenible

García Perales, R. (2012). La educación desde la perspectiva de género. Revista de la Facultad de Educación de Albacete, 1-18.

Ministerio de Educación de El Salvador (MINED). (2017). Resultados de la información estadística de instituciones de educación superior 2016. San Salvador: Ministerio de Educación.

PNUD. (s.f.). PNUD El Salvador. Obtenido de Objetivos de Desarrollo Sostenible: http://www. sv.undp.org/content/el_salvador/es/home/post2015/sdg-overview.html

Ronconi, L. (2018). El acceso a la educación desde una mirada igualitaria: la influencia del derecho internacional de los derechos humanos. Anuario Mexicano de Derecho Internacional, 191211.

Ruiz Fernández, A. R. (2014). Acceso igualitario de las mujeres a la educación en América Latina y el Caribe. Avances y retrocesos. En C. Chacartegui, S. Cuentas, A. R. Ruiz, \& M. E. Blandón, El paper de la dona (págs. 83109). Barcelona, Girona, Lleida, Tarragona: Universitat de Girona. Servei de Publicacions. 
Edicions i Publicacions de la Universitat de Lleida. Universitat Pompeu Fabra. Publicacions de la Universitat Rovira i Virgili.

Secretaría Técnica y de Planificación. (14 de Marzo de 2018). Secretaría Técnica y de Planificación. Obtenido de Socios para el desarrollo conocen avances en la Agenda Nacional de Desarrollo Sostenible: http://www. secretariatecnica.gob.sv/tag/ods/

Sostenibilidad para todos. (s.f.). Sostenibilidad para todos. Obtenido de ¿que son los objetivos de desarrollo sostenible?: https://www. sostenibilidad.com/desarrollo-sostenible/queson-los-objetivos-de-desarrollo-sostenible/
UNESCO. (1998). Conferencia Mundial sobre la Educación Superior. La educación superior en el siglo XXI. Visión y acción. Tomo I Informe Final. París: UNESCO.

UNESCO. (2009). Indicadores de la educación. Especificaciones técnicas. Montreal: UNESCO. 


\section{ANEXOS}

Tabla n. ${ }^{\circ} \mathbf{1}$

Matrícula estudiantil en universidades desagregada por sexo 2012-2016

\begin{tabular}{|c|c|c|c|c|c|c|}
\hline & UNIVERSIDAD // AÑO & MASC.12 & FEM.12 & 2012 & MASC.13 & FEM.13 \\
\hline 1 & ALBERT EINSTEIN & 369 & 156 & 525 & 360 & 188 \\
\hline 2 & AUTÓNOMA DE SANTA ANA & 386 & 839 & 1,225 & 433 & 933 \\
\hline 3 & CAPITÁN GENERAL GERARDO BARRIOS ( * ) & 3,320 & 3,153 & 6,473 & 3,687 & 3,356 \\
\hline 4 & CATÓLICA DE EL SALVADOR ( A*) & 2,381 & 2,832 & 5,213 & 2,565 & 3,035 \\
\hline 5 & CENTROAMERICANA JOSÉ SIMEÓN CAÑAS- UCA ( A ) & 4,395 & 4,527 & 8,922 & 4,136 & 4,309 \\
\hline 6 & CRISTIANA DE LAS ASAMBLEAS DE DIOS & 349 & 406 & 755 & 381 & 432 \\
\hline 7 & DE EL SALVADOR ( $\left.\mathrm{P}^{*}\right)$ & 21,567 & 25,232 & 46,799 & 22,258 & 25,954 \\
\hline 8 & DE ORIENTE ( A ) & 1,831 & 2,215 & 4,046 & 1,888 & 2,231 \\
\hline 9 & DE SONSONATE & 1,509 & 1,407 & 2,916 & 1,465 & 1,354 \\
\hline 10 & DON BOSCO- (UDB) ( A ) & 3,563 & 2,322 & 5,885 & 4,021 & 2,546 \\
\hline 11 & DR. ANDRÉS BELLO ( *) & 2,526 & 6,097 & 8,623 & 2,857 & 6,786 \\
\hline 12 & DR. JOSÉ MATÍAS DELGADO ( A ) & 3,261 & 3,726 & 6,987 & 3,471 & 4,137 \\
\hline 13 & EVANGÉLICA DE EL SALVADOR ( A ) & 1,345 & 2,228 & 3,573 & 1,355 & 2,276 \\
\hline 14 & 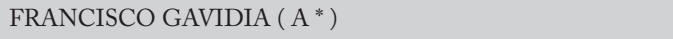 & 6,010 & 6,564 & 12,574 & 6,137 & 6,719 \\
\hline 15 & LUTERANA SALVADOREÑA ( *) & 715 & 1,044 & 1,759 & 808 & 1,136 \\
\hline 16 & MODULAR ABIERTA ( * ) & 2,010 & 3,012 & 5,022 & 2,140 & 3,208 \\
\hline 17 & MONSEÑOR OSCAR ARNULFO ROMERO & 538 & 700 & 1,238 & 588 & 713 \\
\hline 18 & NUEVA SAN SALVADOR & 285 & 312 & 597 & 309 & 373 \\
\hline 19 & PANAMERICANA ( *) & 993 & 1,301 & 2,294 & 1,079 & 1,327 \\
\hline 20 & PEDAGÓGICA DE EL SALVADOR & 2,009 & 3,856 & 5,865 & 1,982 & 3,731 \\
\hline 21 & POLITÉCNICA DE EL SALVADOR & 663 & 278 & 941 & 712 & 286 \\
\hline 22 & SALVADOREÑA ALBERTO MASFERRER ( A ) & 1,274 & 2,178 & 3,452 & 1,331 & 2,410 \\
\hline 23 & TÉCNICA LATINOAMERICANA & 384 & 119 & 503 & 437 & 124 \\
\hline \multirow[t]{3}{*}{24} & TECNOLÓGICA DE EL SALVADOR ( A ) & 10,363 & 9,504 & 19,867 & 10,666 & 9,948 \\
\hline & TOTAL UNIVERSIDADES & 72,046 & 84,008 & 156,054 & 75,066 & 87,512 \\
\hline & PORCENTAJE & $46.17 \%$ & $53.83 \%$ & $100.00 \%$ & $46.17 \%$ & $53.83 \%$ \\
\hline
\end{tabular}

$\left({ }^{*}\right)$ : Incluye a estudiantes de centros regionales.

(A): Institución Acreditada o Reacreditada.

(P): Institución Pública.

Nota: Para el 2014 los 163,607 estudiantes, incluye los 52 estudiantes inscritos en carreras cotituladas en UCA y UDB ( 40 hombres y 12 mujeres).

Fuente: elaboración propia a partir de datos del MINED (2017). 


\begin{tabular}{|c|c|c|c|c|c|c|c|c|c|}
\hline 2013 & MASC.14 & FEM.14 & 2014 & MASC.15 & FEM.15 & 2015 & MASC.16 & FEM.16 & 2016 \\
\hline 548 & 283 & 184 & 467 & 277 & 164 & 441 & 272 & 151 & 423 \\
\hline 1,366 & 472 & 1,037 & 1,509 & 517 & 1,098 & 1,615 & 516 & 1,135 & 1,651 \\
\hline 7,043 & 3,746 & 3,467 & 7,213 & 4,060 & 3,671 & 7,731 & 4,105 & 3,904 & 8,009 \\
\hline 5,600 & 2,797 & 3,374 & 6,171 & 2,988 & 3,699 & 6,687 & 3,107 & 3,863 & 6,970 \\
\hline 8,445 & 3,965 & 4,119 & 8,084 & 3,909 & 4,052 & 7,961 & 3,883 & 4,070 & 7,953 \\
\hline 813 & 322 & 363 & 685 & 294 & 329 & 623 & 279 & 304 & 583 \\
\hline 48,212 & 21,788 & 25,464 & 47,252 & 21,554 & 25,383 & 46,937 & 21,360 & 24,919 & 46,279 \\
\hline 4,119 & 2,035 & 2,456 & 4,491 & 2,159 & 2,757 & 4,916 & 2,331 & 3,042 & 5,373 \\
\hline 2,819 & 1,404 & 1,348 & 2,752 & 1,451 & 1,339 & 2,790 & 1,457 & 1,299 & 2,756 \\
\hline 6,567 & 4,340 & 2,856 & 7,196 & 4,656 & 3,236 & 7,892 & 5,018 & 3,491 & 8,509 \\
\hline 9,643 & 2,736 & 6,711 & 9,447 & 2,703 & 6,834 & 9,537 & 2,813 & 6,952 & 9,765 \\
\hline 7,608 & 3,551 & 4,221 & 7,772 & 3,472 & 4,190 & 7,662 & 3,346 & 4,094 & 7,440 \\
\hline 3,631 & 1,413 & 2,614 & 4,027 & 1,585 & 2,834 & 4,419 & 1,578 & 2,985 & 4,563 \\
\hline 12,856 & 6,013 & 6,610 & 12,623 & 6,067 & 6,504 & 12,571 & 5,705 & 6,278 & 11,983 \\
\hline 1,944 & 890 & 1,171 & 2,061 & 924 & 1,226 & 2,150 & 1,025 & 1,306 & 2,331 \\
\hline 5,348 & 2,199 & 3,177 & 5,376 & 2,305 & 3,255 & 5,560 & 2,323 & 3,534 & 5,857 \\
\hline 1,301 & 596 & 710 & 1,306 & 624 & 713 & 1,337 & 603 & 677 & 1,280 \\
\hline 682 & 311 & 382 & 693 & 329 & 402 & 731 & 322 & 462 & 784 \\
\hline 2,406 & 1,117 & 1,386 & 2,503 & 1,149 & 1,530 & 2,679 & 1,172 & 1,632 & 2,804 \\
\hline 5,713 & 1,794 & 3,235 & 5,029 & 1,787 & 3,253 & 5,040 & 1,761 & 3,313 & 5,074 \\
\hline 998 & 719 & 284 & 1,003 & 717 & 276 & 993 & 732 & 308 & 1,040 \\
\hline 3,741 & 1,466 & 2,680 & 4,146 & 1,627 & 3,021 & 4,648 & 1,679 & 3,128 & 4,807 \\
\hline 561 & 482 & 145 & 627 & 547 & 169 & 716 & 587 & 189 & 776 \\
\hline 20,614 & 10,850 & 10,272 & 21,122 & 10,787 & 10,203 & 20,990 & 10,710 & 10,298 & 21,008 \\
\hline 162,578 & 75,329 & 88,278 & 163,607 & 76,488 & 90,138 & 166,626 & 76,684 & 91,334 & 168,018 \\
\hline $100.00 \%$ & $46.04 \%$ & $53.96 \%$ & $100.00 \%$ & $45.90 \%$ & $54.10 \%$ & $100.00 \%$ & $45.64 \%$ & $54.36 \%$ & $100.00 \%$ \\
\hline
\end{tabular}


Tabla n. ${ }^{\circ} 2$

Matrícula estudiantil en institutos especializados desagregada por sexo 2012-2016

\begin{tabular}{|c|c|c|c|c|c|c|}
\hline & INSTITUTOS ESPECIALIZADOS // AÑO & MASC.12 & FEM.12 & 2012 & MASC.13 & FEM.13 \\
\hline 1 & DE EDUCACIÓN SUPERIOR EL ESPÍRITU SANTO & 113 & 443 & 556 & 88 & 449 \\
\hline 2 & $\begin{array}{l}\text { DE EDUC. SUPERIOR PARA LA FORMACIÓN } \\
\text { DIPLOMÁTICA (P) }\end{array}$ & 9 & 13 & 22 & 9 & 13 \\
\hline 3 & DE PROFESIONALES DE LA SALUD DE EL SALVADOR (*) & 389 & 2,245 & 2,634 & 417 & 2,337 \\
\hline 4 & ESCUELA DE COMUNICACIÓN MÓNICA HERRERA (A) & 115 & 262 & 377 & 118 & 270 \\
\hline 5 & $\begin{array}{l}\text { ESCUELA ESPECIALIZADA EN INGENIERÍA } \\
\text { ITCA-FEPADE (A,P*) }\end{array}$ & 4,314 & 1,500 & 5,814 & 4,034 & 1,387 \\
\hline 6 & ESCUELA SUPERIOR DE ECONOMÍA Y NEGOCIOS (A) & 411 & 328 & 739 & 440 & 331 \\
\hline 7 & ESCUELA SUP. FRANCISCANA ESPECIALIZADA / AGAPE (P) & 443 & 220 & 663 & 429 & 209 \\
\hline 8 & DE NIV. SUP. ACADEMIA NAC. DE SEGURIDAD PÚBLICA (P) & NA & NA & NA & NA & NA \\
\hline 9 & $\begin{array}{l}\text { ESCUELA MILITAR CAPITÁN GENERAL GERARDO } \\
\text { BARRIOS (P) }\end{array}$ & 352 & 36 & 388 & 384 & 34 \\
\hline 10 & $\begin{array}{l}\text { SUPERIOR CENTRO CULTURAL SALVADOREÑO } \\
\text { AMERICANO }\end{array}$ & 69 & 132 & 201 & 77 & 136 \\
\hline 11 & SUPERIOR DE ECONOMÍA Y ADMÓN. DE EMPRESAS (A) & 149 & 114 & 263 & 147 & 125 \\
\hline & TOTAL INSTITUTOS ESPECIALIZADOS & 6,364 & 5,293 & 11,657 & 6,143 & 5,291 \\
\hline & PORCENTAJE & $54.59 \%$ & $45.41 \%$ & $100.00 \%$ & $53.73 \%$ & $46.27 \%$ \\
\hline \multicolumn{7}{|c|}{$\begin{array}{l}\left({ }^{*}\right) \text { : Incluye a estudiantes de centros regionales. } \\
\text { (A): Institución Acreditada o Reacreditada. } \\
\text { (P): Institución Pública. }\end{array}$} \\
\hline
\end{tabular}

Fuente: elaboración propia a partir de datos del MINED (2017).

Tabla n. ${ }^{\circ} 3$

Matricula estudiantil en institutos tecnológicos desagregada por sexo 2012-2016

\begin{tabular}{c|l|c|c|c|c|c|c}
\hline & INSTITUTOS TECNOLÓGICOS // AÑO & MASC.12 & FEM.12 & $\mathbf{2 0 1 2}$ & MASC.13 & FEM.13 & \\
\hline 1 & AMERICANO DE EDUCACIÓN SUPERIOR & 47 & 28 & 75 & 40 & 26 & \\
\hline 2 & DE CHALATENANGO (P) & 305 & 285 & 590 & 272 & 212 & \\
\hline 3 & DE USULUTÁN ( P) & 162 & 101 & 263 & 160 & 96 & \\
\hline 4 & $\begin{array}{l}\text { ESCUELA NAC. DE AGRICULTURA ROBERTO } \\
\text { QUIÑ́NEZ ( P) }\end{array}$ & 304 & 57 & 361 & 274 & 56 & \\
\hline 5 & ESCUELA TÉCNICA PARA LA SALUD & 119 & 682 & 801 & 127 & 696 & \\
\hline 6 & PADRE SEGUNDO MONTES & 29 & 30 & 59 & 43 & 49 & \\
\hline & TOTAL INSTITUTOS TECNOLÓGICOS & $\mathbf{9 6 6}$ & $\mathbf{1 , 1 8 3}$ & $\mathbf{2 , 1 4 9}$ & $\mathbf{9 1 6}$ & $\mathbf{1 , 1 3 5}$ & \\
\hline & PORCENTAJE & $\mathbf{4 4 . 9 5 \%}$ & $\mathbf{5 5 . 0 5 \%}$ & $\mathbf{1 0 0 . 0 0 \%}$ & $\mathbf{4 4 . 6 6 \%}$ & $\mathbf{5 5 . 3 4 \%}$ & \\
\hline
\end{tabular}

(P): Institución Pública.

Fuente: elaboración propia a partir de datos del MINED (2017). 


\begin{tabular}{|c|c|c|c|c|c|c|c|c|c}
\hline $\mathbf{2 0 1 3}$ & MASC.14 & FEM.14 & $\mathbf{2 0 1 4}$ & MASC.15 & FEM.15 & $\mathbf{2 0 1 5}$ & MASC.16 & FEM.16 & $\mathbf{2 0 1 6}$ \\
\hline 537 & 86 & 416 & 502 & 71 & 389 & 460 & 68 & 367 & 435 \\
\hline 22 & 8 & 10 & 18 & 4 & 4 & 8 & 14 & 12 & 26 \\
\hline 2,754 & 414 & 2,153 & 2,567 & 433 & 2,035 & 2,468 & 435 & 1,945 & 2,380 \\
\hline 388 & 124 & 292 & 416 & 138 & 303 & 441 & 147 & 314 & 461 \\
\hline 5,421 & 3,822 & 1,330 & 5,152 & 3,838 & 1,406 & 5,244 & 3,965 & 1,429 & 5,394 \\
& & & & & & & & & \\
\hline 771 & 438 & 335 & 773 & 417 & 366 & 783 & 403 & 388 & 791 \\
\hline 638 & 425 & 190 & 615 & 468 & 247 & 715 & 470 & 272 & 742 \\
\hline NA & NA & NA & NA & NA & NA & NA & 101 & 18 & 119 \\
\hline 418 & 379 & 36 & 415 & 368 & 44 & 412 & 357 & 50 & 407 \\
\hline 213 & 63 & 124 & 187 & 83 & 140 & 223 & 89 & 153 & 242 \\
\hline 272 & 150 & 116 & 266 & 151 & 127 & 278 & 171 & 129 & 300 \\
\hline $\mathbf{1 1 , 4 3 4}$ & $\mathbf{5 , 9 0 9}$ & $\mathbf{5 , 0 0 2}$ & $\mathbf{1 0 , 9 1 1}$ & $\mathbf{5 , 9 7 1}$ & $\mathbf{5 , 0 6 1}$ & $\mathbf{1 1 , 0 3 2}$ & $\mathbf{6 , 2 2 0}$ & $\mathbf{5 , 0 7 7}$ & $\mathbf{1 1 , 2 9 7}$ \\
\hline $\mathbf{1 0 0 . 0 0} \%$ & $\mathbf{5 4 . 1 6 \%}$ & $\mathbf{4 5 . 8 4 \%}$ & $\mathbf{1 0 0 . 0 0 \%}$ & $\mathbf{5 4 . 1 2 \%}$ & $\mathbf{4 5 . 8 8 \%}$ & $\mathbf{1 0 0 . 0 0 \%}$ & $\mathbf{5 5 . 0 6 \%}$ & $\mathbf{4 4 . 9 4 \%}$ & $\mathbf{1 0 0 . 0 0 \%}$ \\
\hline & & & & & & & & \\
\end{tabular}

\begin{tabular}{|c|c|c|c|c|c|c|c|c|c}
\hline $\mathbf{2 0 1 3}$ & MASC.14 & FEM.14 & $\mathbf{2 0 1 4}$ & MASC.15 & FEM.15 & $\mathbf{2 0 1 5}$ & MASC.16 & FEM.16 & $\mathbf{2 0 1 6}$ \\
\hline 66 & 30 & 13 & 43 & 29 & 17 & 46 & 23 & 20 & 43 \\
\hline 484 & 223 & 180 & 403 & 298 & 178 & 476 & 302 & 183 & 485 \\
\hline 256 & 122 & 83 & 205 & 105 & 93 & 198 & 64 & 67 & 131 \\
\hline 330 & 250 & 63 & 313 & 237 & 70 & 307 & 233 & 69 & 302 \\
\hline 823 & 116 & 596 & 712 & 103 & 549 & 652 & 105 & 499 & 604 \\
\hline 92 & 47 & 52 & 99 & 32 & 27 & 59 & 49 & 26 & 75 \\
\hline $\mathbf{2 , 0 5 1}$ & $\mathbf{7 8 8}$ & $\mathbf{9 8 7}$ & $\mathbf{1 , 7 7 5}$ & $\mathbf{8 0 4}$ & $\mathbf{9 3 4}$ & $\mathbf{1 , 7 3 8}$ & $\mathbf{7 7 6}$ & $\mathbf{8 6 4}$ & $\mathbf{1 , 6 4 0}$ \\
\hline $\mathbf{1 0 0 . 0 0 \%}$ & $\mathbf{4 4 . 3 9 \%}$ & $\mathbf{5 5 . 6 1 \%}$ & $\mathbf{1 0 0 . 0 0 \%}$ & $\mathbf{4 6 . 2 6 \%}$ & $\mathbf{5 3 . 7 4 \%}$ & $\mathbf{1 0 0 . 0 0 \%}$ & $\mathbf{4 7 . 3 2 \%}$ & $\mathbf{5 2 . 6 8 \%}$ & $\mathbf{1 0 0 . 0 0 \%}$ \\
\hline
\end{tabular}


Tabla n. ${ }^{\circ} 4$

Matrícula estudiantil en educación superior por área de formación desagregada por sexo 2012-2016

\begin{tabular}{c|l|c|c|c|c|c|c}
\hline & ÁREA DE FORMACIÓN // AÑO & MASC.12 & FEM.12 & $\mathbf{2 0 1 2}$ & MASC.13 & FEM.13 & \\
\hline 1 & ARTE Y ARQUITECTURA & 3,737 & 3,134 & 6,871 & 3,931 & 3,418 & \\
\hline 2 & ECONOMÍA, ADMINISTRACIÓN Y COMERCIO & 18,202 & 25,030 & 43,232 & 18,672 & 25,664 & \\
\hline 3 & SALUD & 8,602 & 21,849 & 30,451 & 9,375 & 23,682 & \\
\hline 4 & CIENCIAS & 1,379 & 1,690 & 3,069 & 1,450 & 1,759 & \\
\hline 5 & AGROPERCUARIA Y MEDIO AMBIENTE & 1,947 & 907 & 2,854 & 2,135 & 1,022 & \\
\hline 6 & DERECHO & 6,955 & 8,713 & 15,668 & 7,115 & 8,909 & \\
\hline 7 & HUMANIDADES & 3,835 & 5,127 & 8,962 & 4,218 & 5,771 & \\
\hline 8 & TECNOLOGÍA & 26,425 & 8,021 & 34,446 & 27,003 & 7,837 & \\
\hline 9 & EDUCACIÓN & 5,306 & 11,041 & 16,347 & 4,999 & 10,649 & \\
\hline 10 & CIENCIAS SOCIALES & 2,988 & 4,972 & 7,960 & 3,227 & 5,227 & \\
\hline & TOTAL GENERAL & $\mathbf{7 9 , 3 7 6}$ & $\mathbf{9 0 , 4 8 4}$ & $\mathbf{1 6 9 , 8 6 0}$ & $\mathbf{8 2 , 1 2 5}$ & $\mathbf{9 3 , 9 3 8}$ & \\
\hline & PORCENTAJE & $\mathbf{4 6 . 3 8 \%}$ & $\mathbf{5 3 . 6 2} \%$ & $\mathbf{1 0 0 . 0 0 \%}$ & $\mathbf{4 6 . 7 3 \%}$ & $\mathbf{5 3 . 2 7 \%}$ & \\
\hline
\end{tabular}

Fuente: elaboración propia a partir de datos del MINED (2017).

Tabla n. ${ }^{\circ} 5$

Graduados de educación superior por área de formación desagregada por sexo 2012-2016

\begin{tabular}{c|l|c|c|c|c|c|c}
\hline & ÁREA DE FORMACIÓN // AÑO & MASC.12 & FEM.12 & $\mathbf{2 0 1 2}$ & MASC.13 & FEM.13 & \\
\hline 1 & ARTE Y ARQUITECTURA & 312 & 325 & 637 & 307 & 305 & \\
\hline 2 & ECONOMÍA, ADMINISTRACIÓN Y COMERCIO & 1,855 & 2,989 & 4,844 & 2,169 & 3,462 & \\
\hline 3 & SALUD & 868 & 3,012 & 3,880 & 887 & 3,139 & \\
\hline 4 & CIENCIAS & 90 & 123 & 213 & 98 & 141 & \\
\hline 5 & AGROPERCUARIA Y MEDIO AMBIENTE & 224 & 82 & 306 & 266 & 87 & \\
\hline 6 & DERECHO & 746 & 1,109 & 1,855 & 796 & 1,090 & \\
\hline 7 & HUMANIDADES & 209 & 268 & 477 & 191 & 296 & \\
\hline 8 & TECNOLOGÍA & 3,241 & 1,212 & 4,453 & 3,677 & 1,334 & \\
\hline 9 & EDUCACIÓN & 1,222 & 2,910 & 4,132 & 1,560 & 2,852 & \\
\hline 10 & CIENCIAS SOCIALES & 284 & 585 & 869 & 304 & 658 & \\
\hline & TOTAL GENERAL & $\mathbf{9 , 0 5 1}$ & $\mathbf{1 2 , 6 1 5}$ & $\mathbf{2 1 , 6 6 6}$ & $\mathbf{1 0 , 2 5 5}$ & $\mathbf{1 3 , 3 6 4}$ & \\
\hline & PORCENTAJE & $\mathbf{4 1 . 2 7 \%}$ & $\mathbf{5 8 . 7 3 \%}$ & $\mathbf{1 0 0 . 0 0 \%}$ & $\mathbf{4 1 . 7 8 \%}$ & $\mathbf{5 8 . 2 2 \%}$ & \\
\hline
\end{tabular}

Fuente: elaboración propia a partir de datos del MINED (2017). 


\begin{tabular}{c|c|c|c|c|c|c|c|c|c|c}
\hline $\mathbf{2 0 1 3}$ & MASC.14 & FEM.14 & $\mathbf{2 0 1 4}$ & MASC.15 & FEM.15 & $\mathbf{2 0 1 5}$ & MASC.16 & FEM.16 & $\mathbf{2 0 1 6}$ \\
\hline 7,349 & 3,979 & 3,759 & 7,738 & 4,083 & 3,960 & 8,043 & 4,056 & 4,171 & 8,227 \\
\hline 44,336 & 18,921 & 25,824 & 44,745 & 19,437 & 26,252 & 45,689 & 19,591 & 26,592 & 46,183 \\
\hline 33,057 & 9,623 & 24,276 & 33,899 & 10,092 & 25,244 & 35,336 & 10,418 & 26,242 & 36,660 \\
\hline 3,209 & 1,481 & 1,742 & 3,223 & 1,503 & 1,830 & 3,333 & 1,459 & 1,776 & 3,235 \\
\hline 3,157 & 2,261 & 1,145 & 3,406 & 2,356 & 1,268 & 3,624 & 2,401 & 1,464 & 3,865 \\
\hline 16,024 & 6,888 & 8,830 & 15,718 & 6,718 & 8,964 & 15,682 & 6,523 & 8,919 & 15,442 \\
\hline 9,989 & 4,382 & 6,149 & 10,531 & 4,561 & 6,466 & 11,027 & 4,538 & 6,437 & 10,975 \\
\hline 34,840 & 26,406 & 7,493 & 33,899 & 26,683 & 7,493 & 34,176 & 27,035 & 7,694 & 34,729 \\
\hline 15,648 & 4,730 & 9,570 & 14,300 & 4,323 & 9,021 & 13,344 & 4,018 & 8,178 & 12,196 \\
\hline 8,454 & 3,355 & 5,479 & 8,834 & 3,507 & 5,635 & 9,142 & 3,641 & 5,802 & 9,443 \\
\hline $\mathbf{1 7 6 , 0 6 3}$ & $\mathbf{8 2 , 0 2 6}$ & $\mathbf{9 4 , 2 6 7}$ & $\mathbf{1 7 6 , 2 9 3}$ & $\mathbf{8 3 , 2 6 3}$ & $\mathbf{9 6 , 1 3 3}$ & $\mathbf{1 7 9 , 3 9 6}$ & $\mathbf{8 3 , 6 8 0}$ & $\mathbf{9 7 , 2 7 5}$ & $\mathbf{1 8 0 , 9 5 5}$ \\
\hline $\mathbf{1 0 0 . 0 0 \%}$ & $\mathbf{4 6 . 5 3 \%}$ & $\mathbf{5 3 . 4 7 \%}$ & $\mathbf{1 0 0 . 0 0 \%}$ & $\mathbf{4 6 . 4 1 \%}$ & $\mathbf{5 3 . 5 9 \%}$ & $\mathbf{1 0 0 . 0 0 \%}$ & $\mathbf{4 6 . 2 4 \%}$ & $\mathbf{5 3 . 7 6 \%}$ & $\mathbf{1 0 0 . 0 0 \%}$ \\
\hline
\end{tabular}

\begin{tabular}{c|c|c|c|c|c|c|c|c|c|c}
\hline $\mathbf{2 0 1 3}$ & MASC.14 & FEM.14 & $\mathbf{2 0 1 4}$ & MASC.15 & FEM.15 & $\mathbf{2 0 1 5}$ & MASC.16 & FEM.16 & $\mathbf{2 0 1 6}$ \\
\hline 612 & 312 & 315 & 627 & 320 & 344 & 664 & 286 & 360 & 646 \\
\hline 5,631 & 2,111 & 3,384 & 5,495 & 2,200 & 3,431 & 5,631 & 2,216 & 3,491 & 5,707 \\
\hline 4,026 & 966 & 3,312 & 4,278 & 1,083 & 3,467 & 4,550 & 1,115 & 3,421 & 4,536 \\
\hline 239 & 93 & 125 & 218 & 84 & 103 & 187 & 89 & 104 & 193 \\
\hline 353 & 239 & 91 & 330 & 233 & 106 & 339 & 319 & 97 & 416 \\
\hline 1,886 & 762 & 1,039 & 1,801 & 670 & 904 & 1,574 & 752 & 954 & 1,706 \\
\hline 487 & 226 & 309 & 535 & 239 & 344 & 583 & 305 & 503 & 808 \\
\hline 5,011 & 3,687 & 1,217 & 4,904 & 3,664 & 1,232 & 4,896 & 3,666 & 1,073 & 4,739 \\
\hline 4,412 & 1,441 & 2,711 & 4,152 & 1,192 & 2,545 & 3,737 & 898 & 1,842 & 2,740 \\
\hline 962 & 278 & 576 & 854 & 281 & 552 & 833 & 322 & 591 & 913 \\
\hline $\mathbf{2 3 , 6 1 9}$ & $\mathbf{1 0 , 1 1 5}$ & $\mathbf{1 3 , 0 7 9}$ & $\mathbf{2 3 , 1 9 4}$ & $\mathbf{9 , 9 6 6}$ & $\mathbf{1 3 , 0 2 8}$ & $\mathbf{2 2 , 9 9 4}$ & $\mathbf{9 , 9 6 8}$ & $\mathbf{1 2 , 4 3 6}$ & $\mathbf{2 2 , 4 0 4}$ \\
\hline & $\mathbf{4 3 . 6 1 \%}$ & $\mathbf{5 6 . 3 9 \%}$ & $\mathbf{1 0 0 . 0 0 \%}$ & $\mathbf{4 3 . 3 4 \%}$ & $\mathbf{5 6 . 6 6 \%}$ & $\mathbf{1 0 0 . 0 0 \%}$ & $\mathbf{4 4 . 4 9 \%}$ & $\mathbf{5 5 . 5 1 \%}$ & $\mathbf{1 0 0 . 0 0 \%}$ \\
\hline
\end{tabular}

